\title{
Successful Resection of a Large Rectal Adenoma Using the Transsacral Approach
}

\author{
TAKUYA HORIO, YOSUKE OKA, ICHITARO SHIRATSUCHI, KOUHEI SAISHO, \\ YUUTAROU MIHARA, REIICHIROU KONDO*, TETSUSHI KINUGUSA, \\ JUN AKIBA* AND YOSHITO AKAGI
}

Department of Surgery and *Department of Pathology, Kurume University School of Medicine, Kurume 830-0011 Japan

Received 25 March 2016, accepted 2 October 2016

J-STAGE advance publication 15 March 2017

Edited by MINORU YAGI

\begin{abstract}
Summary: The transsacral approach is not routinely used for treating rectal tumors. We report the case of a 65-year-old man with a large adenoma at the posterior wall of the mid-rectum who was treated via the transsacral approach. The same lesion had been treated using transsacral endoscopic microsurgery 8 years previously. Moreover, 11 years previously he had undergone a laparotomy for bladder cancer, and an Indiana pouch had been constructed. Abdominal computed tomography showed that the pouch was adjacent to the rectum. Therefore, the less-invasive transsacral approach, rather than the transabdominal approach, was chosen for treatment. The lesion was successfully resected, without disturbing the pouch. Histological analysis indicated tubular adenoma, with a small focus of intramucosal adenocarcinoma, and negative margins. Thus, we achieved successful resection of mid-rectal lesions via the transsacral approach, without the morbidity associated with major laparotomy. We suggest that this procedure should be a part of a surgeon's armamentarium.
\end{abstract}

Key words large rectal adenoma, early rectal cancer, recurrence after TEM, transsacral approach

\section{INTRODUCTION}

The management of a large adenoma in the mid or lower rectum is a common therapeutic dilemma for which several techniques have been proposed. These techniques include endoscopic mucosal resection (EMR), endoscopic submucosal dissection (ESD), transanal endoscopic microsurgery (TEM), standard transanal resection, transsacral resection, and low anterior resection (LAR).

The transsacral approach for lesions located in the mid-rectum has been well described by Kraske, and a modified technique has been used for benign rectal problems and certain rectal cancers [1]. However, in these cases, the transsacral approach is still avoided because of the increased risk of complications such as fecal fistula, dysfunction, and poor perineal wound healing [2]. Nevertheless, this procedure is a reasonable surgical alternative for the management of patients with mid-rectal tumors that cannot be managed by other treatment methods.

In the present report, we describe a case of a large rectal adenoma that was treated using the transsacral approach, and emphasize the utility of this procedure for certain patients with mid-rectal tumors.

\section{CASE REPORT}

A 65-year-old man presented with a 3-month history of bleeding during defecation. Colonoscopy

Corresponding Author: Takuya Horio, Department of Surgery, Kurume University School of Medicine, 67 Asahi-machi, Kurume 830-0011, Japan. Tel: +81942-31-7566 Fax: +81-942-34-0709 E-mail: takuya.horio@gmail.com

Abbreviations: AV, anal verge; CT, computed tomography; EMR, endoscopic mucosal resection; ESD, endoscopic submucosal dissection; TEM, transanal endoscopic microsurgery; LAR, low anterior resection. 
showed a laterally spread tumor $45 \mathrm{~mm}$ in diameter, located $10 \mathrm{~cm}$ proximal to the anal verge (AV), which exhibited a $\mathrm{V}_{\mathrm{I}}$ type pit pattern (irregular arrangement and sizes of IIIL and IV type) [3], (Fig. 1). Barium enema examination showed that the tumor was located at the posterior wall of the mid-rectum (Fig. 2). The same lesion had been treated using TEM 8 years previously at another hospital, and therefore local recurrence was suspected. Analysis of a biopsy specimen obtained from the tumor indicated the presence of an adenoma with severe atypia. However, based on the colono-

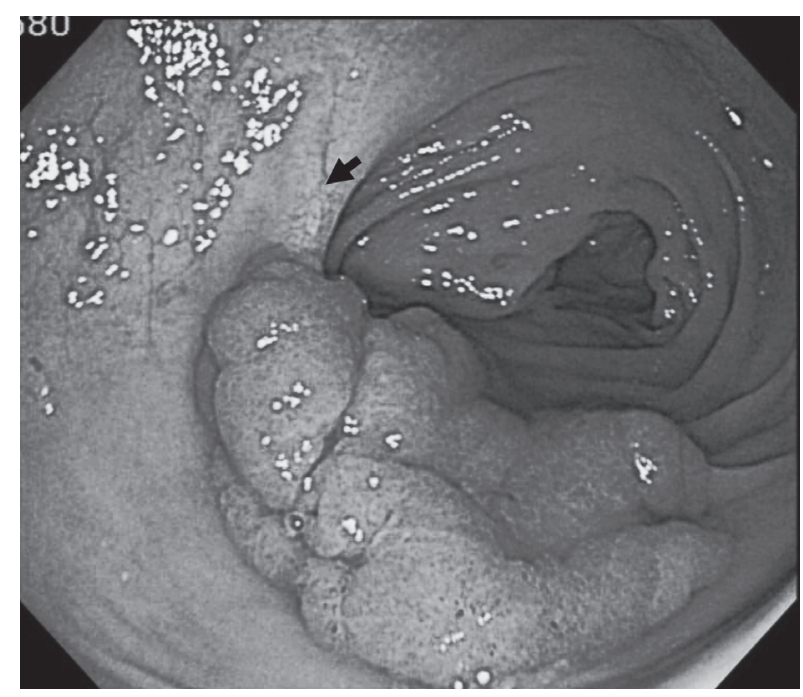

Fig. 1. A colonoscopic examination revealed a large sessile tumor in the rectum, located $10 \mathrm{~cm}$ proximal to the anal verge. The lesion was on the preoperative scar line (arrow).

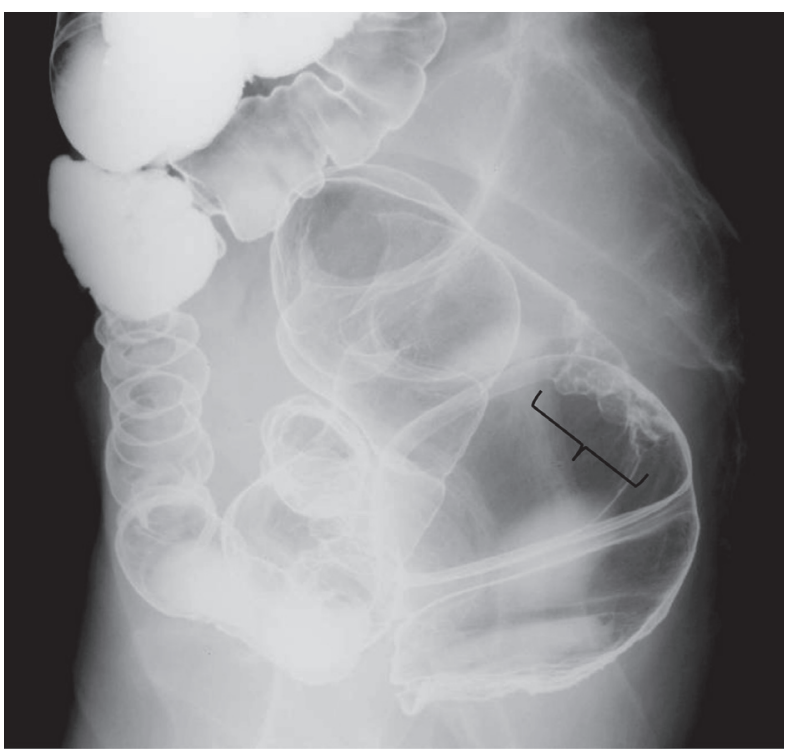

Fig. 2. Barium enema examination showed a tumor, located at posterior wall of the mid-rectum. scopic findings, a part of this tumor was strongly suspected to be a carcinoma.

Furthermore, 11 years previously the patient had undergone a cystectomy, with the creation of an Indiana pouch, for a bladder carcinoma, which had required intermittent self-catheterization 3 times per day. Abdominal computed tomography (CT) indicated that the Indiana pouch was located adjacent to the anterior wall of the rectum in the same slice in which the tumor was noted. Abdominal and pelvic CT did not show any evidence of metastasis, including to the lymph node in the mesorectum (Fig. 3). Based on these findings, we decided to perform surgery via the transsacral approach.

\section{Surgical procedure}

The patient was placed under general anesthesia in the prone jackknife position. Both buttocks were taped to the side of the table to spread the gluteal cleft. A downward skin incision was made from a point located

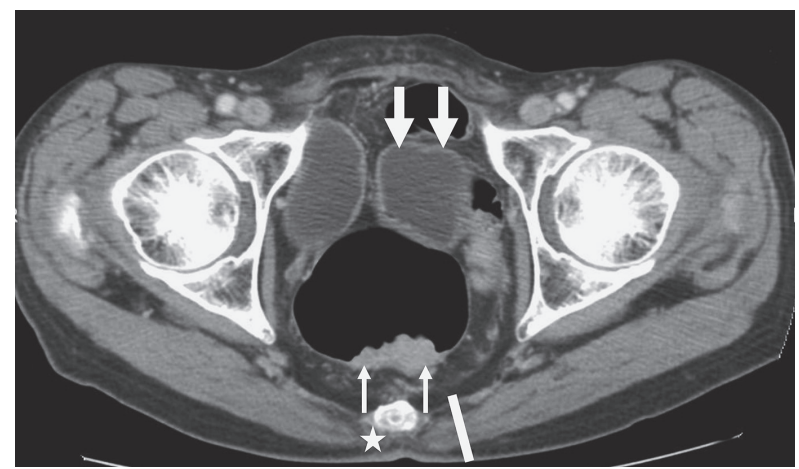

Fig. 3. Abdominal computed tomography revealed the Indiana-pouch (large arrows) adjacent to the rectum in the same slice that showed the tumor. rectal tumor: small arrows sacrum: star approach line: white line

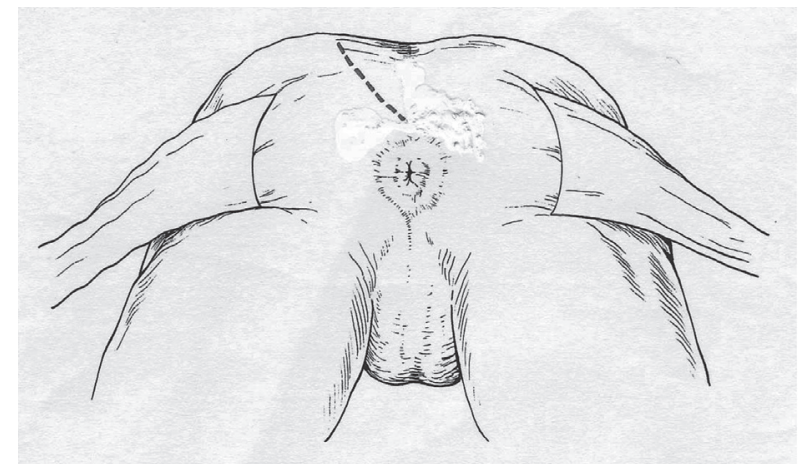

Fig. 4. Position and skin incision line Position: prone jackknife position Skin incision line: dashed line. 


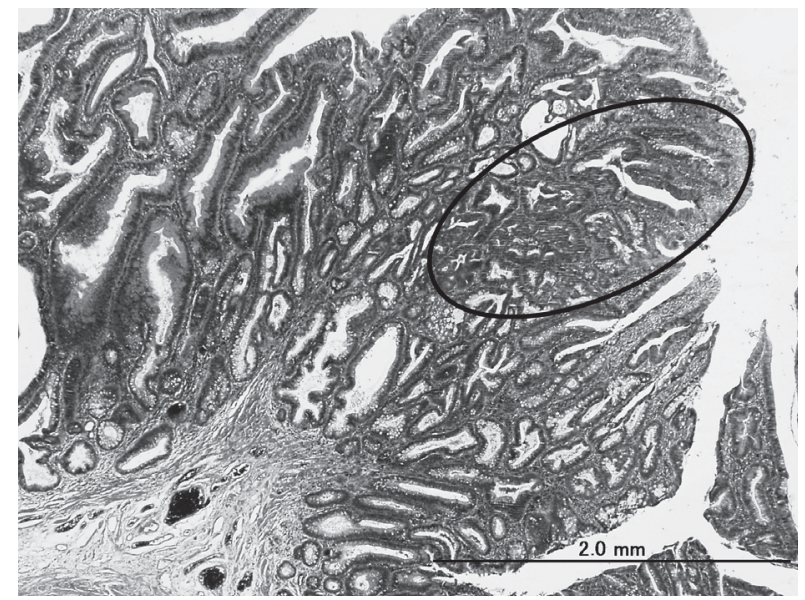

Fig. 5. Histology revealed the tumor was mainly composed of tubular adenoma. A small focus of adenocarcinoma was within the tumor, although these cells did not display any submucosal or lymphvascular invasion. $(\mathrm{H} \& \mathrm{E} \times 20)$.

immediately to the left of the sacrococcygeal junction to the perianal lesion along the sacrum (Fig. 4). The distal coccyx was transected, and the fascia of Waldeyer was opened to expose the perirectal fat. The mesorectum and lower rectum were decorticated to the maximum possible extent. However, the anterior wall of the rectum could not be sufficiently decorticated due to the risk of affecting the pouch. During a digital rectal examination, the lesion was palpated and an incision was made in the lateral wall of the rectum adjacent to the tumor. En-bloc-full-thickness excision of the lesion with the mesorectum was performed under direct vision, while maintaining a sufficient surgical margin. The rectal wall defect was closed in a longitudinal fashion using an Albert-Lembert anastomosis, and a drain was placed in the buttocks. Thus, the lesion was successfully removed by local resection, without touching the pouch.

Histological examination identified the lesion as an intramucosal adenocarcinoma within the adenoma component with negative margins. No evidence of lymphovascular invasion of the tumor was noted (Fig. 5). Although wound infection developed postoperatively, adequate drainage resulted in spontaneous resolution. At 20 months after surgery, the patient was found to be symptom-free without any signs of recurrence or discomfort.

\section{DISCUSSION}

In the present report, we describe the successful en-bloc resection of a large rectal adenoma using the transsacral approach, and thus emphasize the utility of this procedure for certain patients with mid-rectal tumors.

In the present case, we strongly suspected that the large adenoma had a malignant component based on the clinical and colonoscopic findings. Therefore, an en-bloc resection was desirable to enable histologic evaluation. The use of transanal local resection techniques such as EMR, ESD, TEM, and standard transanal excision was avoided as the tumor lesion was located on the scar of a previous surgery, and the technical difficulty of removing the tumor would have increased the risk of piecemeal resection. Moreover, the tumor was large, and the proximal extent of the tumor could only be poorly visualized. The transabdominal approach increased the risk of damaging the pouch because of its adhesion to the rectum. However, because the presacral space had not been affected, we decided to perform transsacral resection, as it appeared to be the safest and most adequate treatment option for this patient.

Histologic evaluation of the entire tumor after enbloc resection is the best method for identifying the presence of a malignant component, evaluating the risk of distant spread, and indicating the need for secondary treatment. EMR did not guarantee complete excision because of the associated technical difficulties and may not have yielded an en-bloc specimen for histopathological examination [4]. ESD has also been used for the treatment of superficial colorectal cancers for the en-bloc removal of lesions larger than $2 \mathrm{~cm}$ to reduce the risk of recurrence for lesions showing $V_{I}$ type pit pattern at high-magnification; for large protruding lesions suspected to be carcinomas, including those with fibrosis due to previous biopsies; and for postEMR residual carcinomas [5]. However, this method is technically difficult, has a long procedure time, and shows a perforation rate that is significantly higher than that of EMR [6]. TEM has been increasingly used for large, sessile rectal adenomas [7,8]. However, this procedure has only gradually gained popularity, because of the associated high cost and steep learning curve [9]. Furthermore, standard transanal excision is curative and less invasive than transsacral resection or LAR; however, resection of tumors distant from the $\mathrm{AV}$ is difficult with this method [9].

The transsacral approach is not widely accepted due to the risk of postoperative complications such as fecal fistula, dysfunction, and poor perineal wound healing [2]. However, most of the studies that reported a high incidence of complications were conducted prior to the introduction of proper bowel preparation, 
prophylactic antibiotics, and adequate drainage techniques $[1,10]$. In fact, this procedure has been recommended by several surgeons for the management of mid-rectal lesions, particularly in cases of frail patients, in whom a laparotomy is associated with unacceptable risks [10,11]. This procedure also presents many advantages, including the possibility of obtaining excellent exposure without the need for laparotomy and the avoidance of the risk of urogenital dysfunction following TEM [12]. This procedure is generally believed to be indicated in the following situations: (1) when the tumor is located $8-10 \mathrm{~cm}$ from the $\mathrm{AV}$, and TEM, EMR or ESD are not feasible; (2) when there is no evidence of metastasis, including lymph node metastasis in the mesorectum; and (3) when LAR may result in overtreatment or laparotomy may be associated with unacceptable risks. After considering the advantages and disadvantages of each technique, transsacral resection was considered as the most appropriate for the management of this case.

In conclusion, the transsacral approach is largely avoided, but can be a useful surgical alternative for the management of patients with mid-rectal tumors that cannot be managed by other treatment options. Knowledge of this approach contributes to a better understanding of the anatomy of the rectum and pelvic organs, and extends the range of therapeutic strategies.

\section{CONFLICTS OF INTEREST}

None of the authors have any conflicts of interest to declare.

\section{REFERENCES}

1. Sweeney WB, and Deshmukh N. Modified Kraske approach for disease of the mid-rectum. Am J Gastroenterol. 1991; 86:75-78.

2. Terkivatan T, den Hoed PT, Lange JF Jr, Koot VC, van Goch $\mathrm{JJ}$ et al. The place of the posterior surgical approach for lesions of the rectum. Dig Surg. 2005; 22:86-90.

3. Tanaka S, Kaltenbach T, Chayama K, and Soetikno R. High magnification at colonoscopy (with videos). Gastrointest Endosc. 2006; 64:604-613.

4. Jameel JK, Pillinger SH, Moncur P, Tsai HH, and Duthie GS. Endoscopic mucosal resection (EMR) in the management of large colorectal polyps. Colorectal Dis. 2006; 8:497-500.

5. Tanaka S, Oka S, and Chayama K. Colorectal endoscopic submucosal dissection: present status and future perspective, including its differentiation from endoscopic mucosal resection. J Gastroenterol. 2008; 43:641-651.

6. Santos CE, Malaman D, and Pereira-Lima JC. Endoscopic mucosal resection in colorectal lesion: a safe and effective procedure even in lesions larger than $2 \mathrm{~cm}$ and in carcinomas. Arq Gastroenterol. 2011; 48:242-247.

7. Ramirez JM, Aguilella V, Gracia JA, Ortego J, Escudero P et al. Local full-thickness excision as first line treatment for sessile rectal adenomas: long-term results. Ann Surg. 2009; 249:225-228.

8. Allaix ME, Arezzo A, Cassoni P, Famiglietti F, and Morino M. Recurrence after transanal endoscopic microsurgery for large rectal adenomas. Surg Endosc. 2012; 26:2594-2600.

9. Otsuji E, Fujiyama J, Takagi T, Ito T, Hagiwara A et al. Transanal excision of a large rectal polyp assisted by transsacral manipulation of the rectum. Dis Colon Rectum. 2004; 47:1420-1422.

10. Kronborg O, Kramhoft J, Baker O, and Sprechler M. Early complications following operations for cancer of the rectum and anus. Dis Colon Rectum. 1974; 17:741-749.

11. Bergamaschi R, and Arnaud JP: Management of large encircling mid-rectal adenomas in frail patients. Int $\mathrm{J}$ Colorectal Dis. 1995; 10:53-54.

12. Onaitis M, Ludwig K, Perez-Tamayo A, Gottfried M, Russell L et al. The Kraske Procedure: a critical analysis of a surgical approach for mid-rectal lesions. J Surg Oncol. 2006; 94:194-202. 